\title{
Midinfrared Dispersion Relations in InP-Based Photonic Crystal Slabs Revealed by Fourier-Transform Angle-Resolved Reflection Spectroscopy
}

\author{
Siti Chalimah, ${ }^{1,2}$ Yuanzhao Yao $\odot,{ }^{1}$ Naoki Ikeda, ${ }^{1}$ Kei Kaneko, ${ }^{3}$ Rei Hashimoto, ${ }^{3}$ Tsutomu Kakuno, ${ }^{3}$ \\ Shinji Saito $\odot,{ }^{3}$ Takashi Kuroda $\odot,{ }^{1,2, *}$ Yoshimasa Sugimoto, ${ }^{1}$ and Kazuaki Sakoda ${ }^{1}$ \\ ${ }^{1}$ National Institute for Materials Science (NIMS), 1-1 Namiki, Tsukuba 305-0044, Japan \\ ${ }^{2}$ Graduate School of Engineering, Kyushu University, NIMS, 1-1 Namiki, Tsukuba 305-0044, Japan \\ ${ }^{3}$ Corporate Manufacturing Engineering Center, Toshiba Corporation, Isogo, Yokohama 235-0017, Japan
}

(Received 2 April 2021; revised 29 May 2021; accepted 3 June 2021; published 30 June 2021)

\begin{abstract}
Photonic crystals (PCs) offer unique ways to control light-matter interactions. The measurement of dispersion relations is a fundamental prerequisite if we are to create various functionalities in PC devices. Angle-resolved spectroscopic techniques are commonly used for characterizing PCs that work in the visible and near-infrared regions. However, the techniques cannot be applied to the mid- and long-wavelength infrared regions due to the limited sensitivity of infrared detectors. Here, we propose an alternative approach to measuring infrared dispersion relations. We construct a high-precision angle-resolved setup compatible with a Fourier-transform spectrometer with an angle resolution as high as $0.3^{\circ}$. Hence, the reflection spectra are mapped to the two-dimensional (2D) photonic band structures of $\operatorname{In}(\mathrm{Ga}, \mathrm{Al}) \mathrm{As} / \mathrm{InP}-$ based PC slabs, which are designed as midinfrared-PC surface-emitting lasers. We identify complex PC modes with the aid of polarization-selection rules derived by group theory. Spectral analysis makes it possible to evaluate the mode quality $(Q)$ factors. Therefore, angle-resolved reflection is a useful way of optimizing 2D PC parameters for midinfrared devices.
\end{abstract}

DOI: 10.1103/PhysRevApplied.15.064076

\section{INTRODUCTION}

Photonic crystal (PC) surface-emitting lasers (PCSELs) are an emerging laser architecture the unique characteristics of which include a high output power that scales with the device area, good mode quality, and low beam divergence due to the high spatial coherence across the entire device [1-4]. The principle of the laser action relies on the zero group velocity of light at photonic band edges, which results in strong in-plane feedback inside a PC slab. Moreover, when band edges are formed at the $\Gamma$ point in momentum space, radiation into free space occurs purely out of plane, i.e., normal to the slab, as a consequence of momentum-conservation rules. Hence, careful PC design that realizes perfect resonance between the band-edge mode and the material gain frequencies is essential for achieving vertical emission. The measurement of in-plane dispersion relations is thus frequently required to verify optimum conditions in fabricated devices.

\footnotetext{
*Corresponding author. kuroda.takashi@nims.go.jp

Published by the American Physical Society under the terms of the Creative Commons Attribution 4.0 International license. Further distribution of this work must maintain attribution to the author(s) and the published article's title, journal citation, and DOI.
}

A technique commonly used to characterize photonic band structures in PCSEL devices is the angle-resolved observation of subthreshold luminescence spectra [57]. Light waves, which are associated with spontaneous emission from gain media and then coupled to in-plane PC modes, are diffracted into free space along particular directions, which conserve wave-vector projections to the slab plane [8]. Hence, the angular profiles of the emission spectra are mapped to two-dimensional (2D) photonic band structures, similar to the electronic band structures revealed by angle-resolved photoelectron spectra (ARPES). The technique is widely used for the characterization of visible and near-infrared wavelength PCSELs. However, the technique cannot be applied to midinfrared wavelengths due to the limited sensitivity of infrared detectors. To develop midinfrared PCSELs, which take advantage of quantum cascade laser (QCL) technologies [9-12], an alternative scheme is required for characterizing PC slabs.

Recently, we reported the use of angle-resolved reflection measurement to determine infrared dispersion relations in model PC slabs based on silicon-on-insulator (SOI) waveguides [13]. We put considerable effort into achieving high angle resolution and developed a highprecision variable-angle reflection apparatus that is compatible with a Fourier transform spectrometer [14]. 
Here, we apply the angle-resolved reflection technique to the characterization of actual PCSEL devices formed of $\mathrm{In}(\mathrm{Ga}, \mathrm{Al}) \mathrm{As} / \mathrm{InP}$-based QCL multilayer structures. We identify complex PC modes in the vicinity of the $\Gamma$ point with the aid of rigorous polarization-selection rules derived by group theory [15]. Moreover, spectral analysis makes it possible to evaluate the quality $(Q)$ factors of waveguide modes semiquantitatively. Thus, the angle-resolved reflection measurement is a useful tool with which to determine fundamental parameters for laser actions.

\section{EXPERIMENTAL PROCEDURE}

\section{A. Samples}

Midinfrared QCL multilayer structures are grown on $n^{+} \operatorname{InP}(100)$ by molecular-beam epitaxy. We grow a lattice-matched (In, Ga)As buffer layer with a thickness of $1.0 \mu \mathrm{m}$, an InP bottom cladding layer with a thickness of $2.5 \mu \mathrm{m}$, and a thin (In, Ga)As guiding layer with a thickness of $0.3 \mu \mathrm{m}$, followed by (In, Ga)As/(In,Al)As strain-compensated multiple quantum wells (MQWs) with a total thickness of $1.6 \mu \mathrm{m}$. The MQWs serve as a QCL active layer that has an optical gain at a center wavelength of $4.387 \mu \mathrm{m}$. We then grow a $1.0-\mu \mathrm{m}$-thick (In, Ga)As layer, which we use for PC processing [for the layer sequence and the expected refractive indices, see Fig. 5(a) in the Appendix].

Finite-element calculations are carried out to determine the target PC design, that is, a 2D square lattice of (In, Ga)As cylindrical pillars with a height of $0.8 \mu \mathrm{m}$, a radius of $0.565 \mu \mathrm{m}$, and a lattice constant $(a)$ of $1.36 \mu \mathrm{m}$. This design results in a resonance between the measured QCL frequency and the second-lowest band edge at the $\Gamma$ point $\left(\Gamma^{(2)}\right)$ in the first Brillouin zone. (The $\Gamma^{(2)}$ band edge originates from zone folding at the $X$ symmetric point.) Here, we deal mainly with the TM-like polarization modes, since a QCL employs intersubband electronic transitions, which have dipole moments perpendicular to the MQW layer.

Three-millimeter-square PC slabs with $a=1.33,1.36$, and $1.39 \mu \mathrm{m}$ are fabricated using electron-beam lithography and reactive ion-beam etching techniques. $\left(\mathrm{SiO}_{2}\right.$ is used as a mask for the dry etching.) Note that the final PCSEL devices have another InP layer on top, which serves as a top cladding layer and then (In, Ga)As pillars are embedded, followed by a metal gate. We study the samples before InP regrowth and metal-gate deposition. The laser action of the final PCSEL devices will be reported in a forthcoming paper [16].

\section{B. Measurement setups}

Midinfrared angle-resolved reflection measurements are performed using a Fourier-transform spectrometer (Jasco, FT/IR-6800) attached to a home-built variable-angle apparatus, where infrared light is carefully collimated and beam divergence is limited to less than $0.3^{\circ}$. The collimated beam passes through an infrared beam splitter, which then transmits the beam from the sample to the detector. The setup enables us to observe reflection spectra even at normal incidence and to change the incident angle $(\theta)$ from $-3.7^{\circ}$ to $+3.7^{\circ}$ across zero. A wire-grid polarizer is inserted into the beam path and it allows us to resolve the $p$ - and $s$-polarized components, which have the electric field parallel and perpendicular to the incident plane, respectively. The design, which is further detailed in Ref. [14], makes it possible to determine the mode frequencies as a function of in-plane wave-vector and dispersion relations in the vicinity of the $\Gamma$ point.

\section{RESULTS AND DISCUSSION}

\section{A. Midinfrared characterization of PC slabs}

Figure 1 shows reflection spectra at normal incidence $(\theta=0)$ for samples with different lattice constants. All the samples exhibit similar spectral curves, which shift to higher wave numbers (shorter wavelengths) in unison for smaller lattice constants. (The onset of noisy spectra at $2320-2375 \mathrm{~cm}^{-1}$, as shown by the gray region, is due to light absorption by $\mathrm{CO}_{2}$.) The observed scaling behavior is evidence that signature spectra are induced by resonant coupling to PC modes.

The vertical arrows seen above each spectrum indicate the mode energies calculated using the finite-element method. The first and second arrows from the left (lowwave-number side) are the lowest-energy TE-like and TM-like modes, respectively. They are denoted by $\mathrm{TE}^{(1)}$

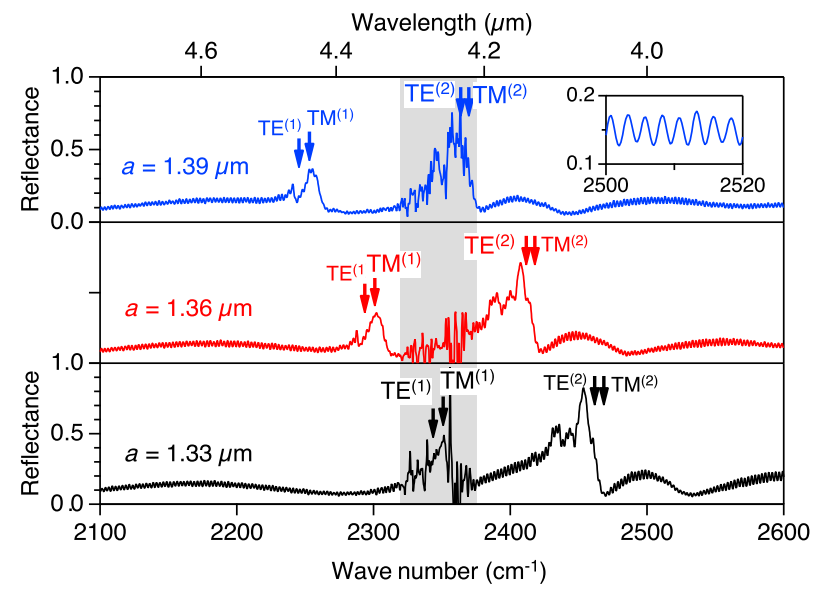

FIG. 1. Normal-incidence reflection spectra for samples with different lattice constants, $a=1.33,1.36$, and $1.39 \mu \mathrm{m}$. The gray region indicates a $\mathrm{CO}_{2}$ absorption band, which causes clear spectral noises. The small sinusoidal modulation superimposed on the spectra is due to Fabry-Perot interference between the top and bottom surfaces of the sample, as highlighted by the expanded spectrum in the inset. 
and $\mathrm{TM}^{(1)}$. The third and fourth arrows are higher-order confinement modes $\left(\mathrm{TE}^{(2)}\right.$ and $\mathrm{TM}^{(2)}$ ), which have a nodal field distribution in a direction normal to the slab (for the field distributions, see Fig. 5 in the Appendix). These calculated modes are in fairly good agreement with the measured spectral peaks, although the peak intensities are strongly dependent on the mode index, as discussed later. A discrepancy between the calculated and the measured mode energies is attributed to the use of inaccurate refractive indices for the constituent materials, particularly that of the bottom (In, Ga)As buffer layer, which contains relatively dense dopants and serves as an antiguiding plasmonic layer.

Figure 2 shows a series of reflection spectra for various incident angles. Here, we study a sample where $a=$ $1.36 \mu \mathrm{m}$ and analyze the spectra for both $s$ and $p$ polarizations. In Figs. 2(a) and 2(b), from bottom to top, the incident beam is tilted from the surface normal toward the [100] in-plane axis, where the wave vector is moved from $\Gamma$ to $X$ in momentum space. For $p$ polarization [Fig. 2(a)],
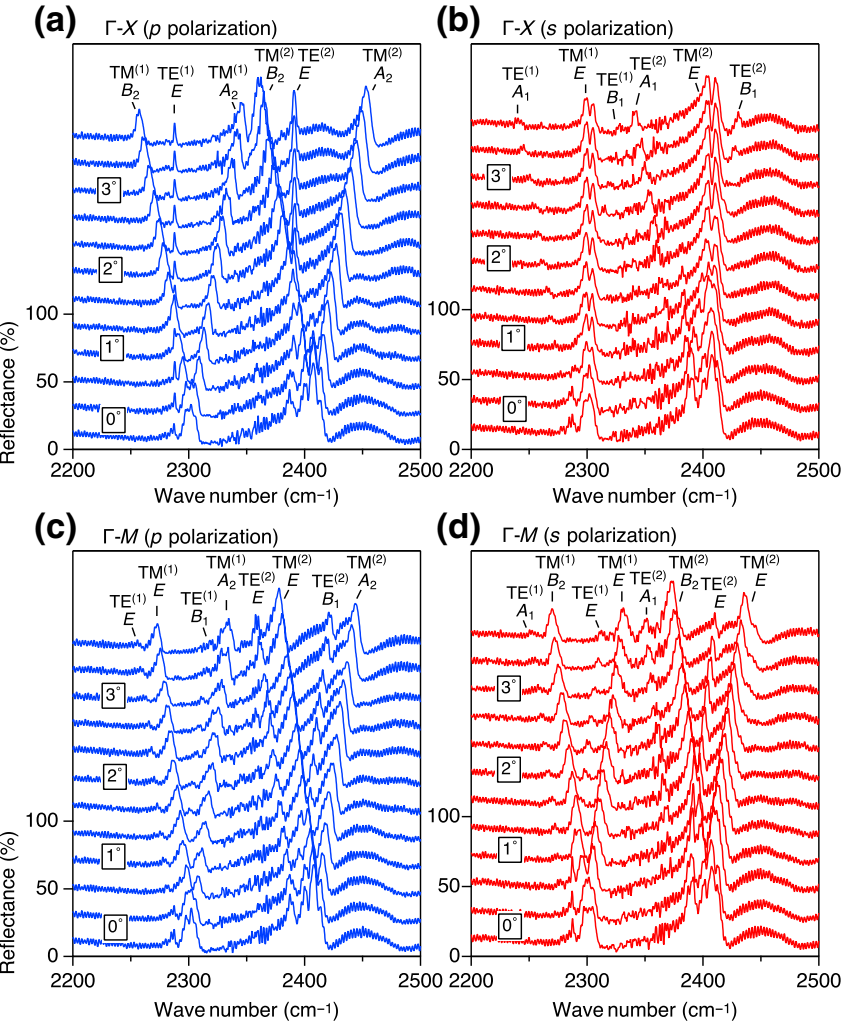

FIG. 2. Angle-resolved reflection spectra for the sample where $a=1.36 \mu \mathrm{m}$. The spectra in each panel are arranged from bottom to top, $\theta=0$ to approximately $3.7^{\circ}$ in $0.35^{\circ}$ steps. (a),(b) The $p$ - and $s$-polarized spectra, respectively, where the incident beam is tilted inside a plane that contains the [100] axis of the square lattice. Accordingly, the wave vector moves along the $\Gamma-X$ axis in the first Brillouin zone. (c),(d) The polarized spectra when the wave vector moves along the $\Gamma-M$ axis. The peak assignments are also indicated at the top in each panel. two intense peaks, which are classified as $\mathrm{TM}^{(1)}$ and $\mathrm{TM}^{(2)}$ at $\theta=0$, are split into two peaks that move to the opposite side. Other small peaks classified as $\mathrm{TE}^{(1)}$ and $\mathrm{TE}^{(2)}$ stay almost constant. In contrast, for $s$ polarization [Fig. 2(b)], the intense peaks do not move but small split peaks, which originate from $\mathrm{TE}^{(1)}$ and $\mathrm{TE}^{(2)}$, are apparent at $\theta \gg 0$.

In Figs. 2(c) and 2(d), the beam is tilted toward the [110] axis ( $45^{\circ}$ away from [100]), and the wave vector is moved from $\Gamma$ to $M$. In this geometry, all the peaks split and shift as the angle $\theta$ increases. Polarization dependence is not evident in these plots but we analyze the significant energy shift between the $s$ - and $p$-polarized spectra. See also the spectral comparison in Fig. 4(a).

\section{B. Mode assignment and dispersion relations}

We assign these reflection peaks in terms of irreducible representations of the $C_{4 v}$ point group [15]. For a uniform slab with no periodic index modulation, the band edge at the $\Gamma^{(2)}$ point has a fourfold degeneracy, which is then lifted into a doubly degenerate $E$ mode and two other nondegenerate modes when the $C_{4 v}$ symmetric modulation is introduced into the slab. The nondegenerate modes are assigned to $A_{1}$ and $B_{1}$ for the TE-like modes and to $A_{2}$ and $B_{2}$ for the TM-like modes, where we consider the symmetry operation of magnetic fields. These mode symmetries are consistent with the prediction by group theory based on the zone folding of TE and TM planar waveguide bands [15]. Of these modes, only the $E$ mode is optically active at normal incidence but the other modes are inactive due to symmetry mismatching. At off-normal incidence, all the modes become active and split. Group theory also predicts polarization-selection rules for the emergence of reflection peaks, which are described in Table I [17]. Using this table, we can safely identify all the measured peaks. The assignment results can be seen at the top of each panel in Fig. 2. It is noteworthy that the above-mentioned symmetries are defined at the $\Gamma$ point and the measured spectral peaks at finite incident angles do not necessarily follow the relevant symmetry characteristics.

We evaluate the center energies of the reflection peaks via fitting. For simplicity, we use a multiple Gaussian function as a fitting model. The extracted energies for

TABLE I. Selection rules for the observation of spectral peaks around the $\Gamma^{(2)}$ band edge in $C_{4 v}$ symmetric PC slabs.

\begin{tabular}{lcc}
\hline \hline Mode $^{\mathrm{a}}$ & $\Gamma-X$ & $\Gamma-M$ \\
\hline$E$ & $s, p$ & $s, p$ \\
$A_{1}$ & $s$ & $s$ \\
$A_{2}$ & $p$ & $p$ \\
$B_{1}$ & $s$ & $p$ \\
$B_{2}$ & $p$ & $s$ \\
\hline \hline
\end{tabular}

${ }^{\text {a }}$ These mode symmetries are defined with the symmetry operation of magnetic fields. 
(a)

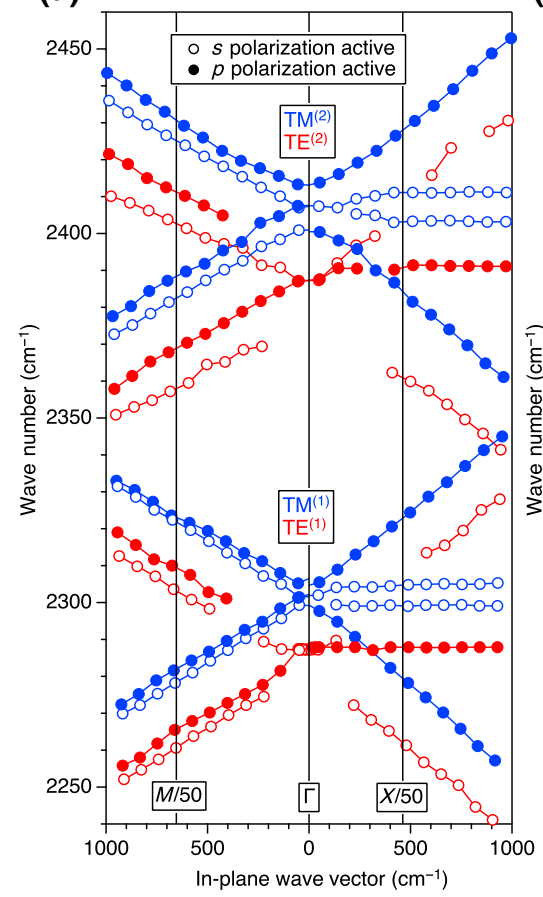

(b)

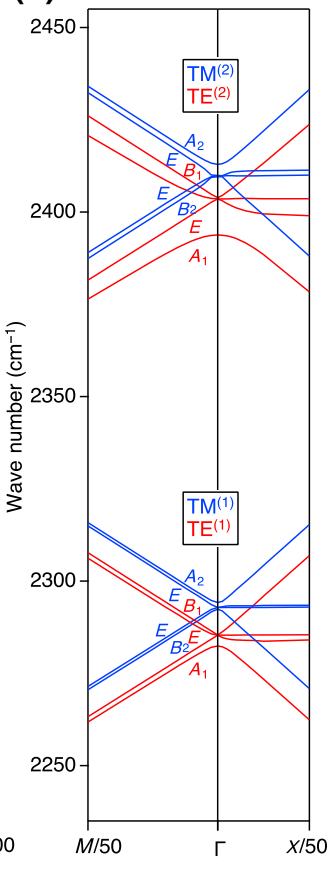

FIG. 3. (a) Dispersion relations based on measured angleresolved reflection spectra in Fig. 2. (b) Calculated 2D dispersion relations, where we assume that $a=1.365 \mu \mathrm{m}$. The abscissa is normalized to $1 / 50$ of the wave vector at the $X$-band edge $(\pi / 50 a)$ and the $M$-band edge $(\sqrt{2} \pi / 50 a)$.

different $\theta$ values are summarized in the band-dispersion curves in Fig. 3(a), where we plot the spectral peak energies (in wave-number units) as a function of a wave-vector projection to the slab plane, i.e., $(2 \pi / \lambda) \sin \theta$, where $\lambda$ is the vacuum wavelength. For comparison, Fig. 3(b) shows calculated band-dispersion curves over $1 / 50$ of the first Brillouin zone. Both the measured and the calculated curves agree well. Moreover, we analyze the field distribution of each mode and confirm that their symmetry agrees with our mode assignment based on the selection rules (see Figs. 6 and 7 and the related discussion in the Appendix). Thus, angle-resolved reflection measurement is a useful technique for experimentally determining photonic band structures.

\section{C. $Q$-factor quantification}

Figure 4(a) compares reflection spectra at $\theta=3.7^{\circ}$ for different geometries and polarizations, where we focus on the $\mathrm{TE}^{(1)}$ and $\mathrm{TM}^{(1)}$ frequency regions. The vertical lines indicate the assigned peak energies, which are determined by fitting. The spectra indicate a strong intensity dependence on the mode index (reflection peaks of different modes have different intensities). We can speculate that the peak intensity is related to the mode coupling strength and thus the inverse of the $Q$ factor, i.e., the rate of energy (a)

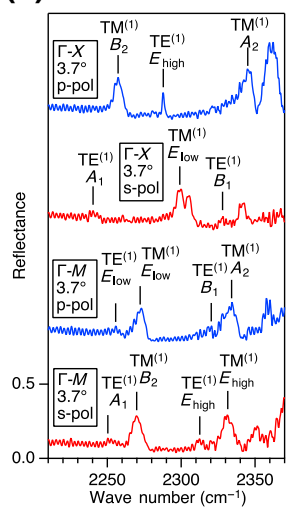

(b)

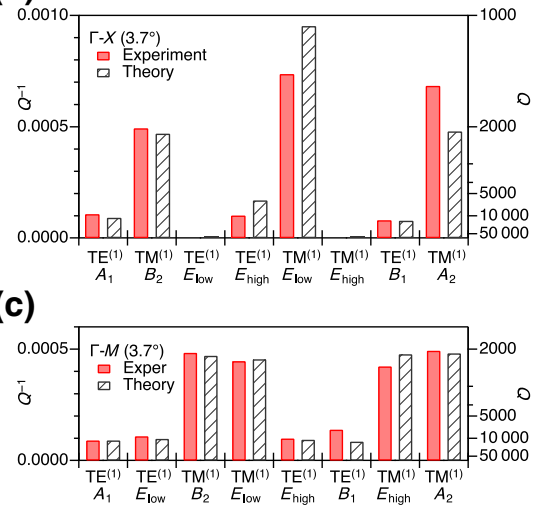

FIG. 4. (a) Reflection spectra at $\theta=3.7^{\circ}$ around the $\mathrm{TE}^{(1)}$ and $\mathrm{TM}^{(1)}$ frequency regions for different geometries and polarizations. The vertical lines indicate the mode center energies, which we determine via fitting. (b),(c) A comparison of the experimentally determined $Q^{-1}$ values (red filled bars) and theoretically obtained $Q^{-1}$ values (black hatched bars) for each mode depicted in (a) with (b) $\Gamma-X$ and (c) $\Gamma-M$ geometries. Note that the experimental $Q^{-1}$ values are proportional to the areas of the reflection peaks.

leakage from the waveguide. The following consideration supports the validity of this speculation.

A rigorous spectral analysis of 2D PC systems proves that the phase of electromagnetic waves transmitted through a PC slab is shifted by $\pi$ across the resonance frequency [18]. Accordingly, the transmittance $|T|^{2}$ goes to zero at resonance and the reflectance $|R|^{2}$ goes to unity, since $|R|^{2}=1-|T|^{2}$ for PC slabs made of nonabsorbing materials. Thus, the area (the spectrally integrated intensity) of the reflection peaks roughly scales with the spectral width $\propto Q^{-1}$ independent of other factors, although the exact spectral shape is rather complicated due to interference between the resonant spectral component and nonresonant backgrounds. In reality, the ideally sharp spectral structures are masked due to the influence of PC inhomogeneities and distributed angles of incidence. Nevertheless, we are able to assume that the peak areas are inversely proportional to the mode $Q$ factors.

Figure 4(b) shows the experimentally determined and theoretically obtained $Q^{-1}$ values for all the $\mathrm{TM}^{(1)}$ and $\mathrm{TE}^{(1)}$ modes at $\theta=3.7^{\circ}$. For the experimental determination we adopt a simple expression, namely, $Q^{-1}=$ $(2 / \pi) S / \omega_{0}$, where $S$ is the peak area, i.e., $\int|R|^{2} d \omega$, and $\omega_{0}$ is the mode frequency. The coefficient $(2 / \pi)$ arises from the normalization factor of the Lorentzian function. The measured $Q^{-1}$ values reveal a fairly good agreement with the theoretical $Q^{-1}$ values, which we calculate using the finite-element method, even without the use of any adjustable parameters. Hence, the reflection peak intensity provides a useful measure that is equivalent to the mode $Q$ factor, i.e., it constitutes key information for understanding lasing properties. 
Throughout this work, we assume that refractive indices are real numbers and ignore the impact of absorption loss on the mode $Q$ factors. Hence, the $Q^{-1}$ values, which we analyze theoretically and experimentally, are governed purely by diffraction (radiation) loss. The $Q^{-1}$ values are therefore equivalent to the rates of energy leakage from the waveguide to free space. To clarify the impact of material absorption on $Q$, we also calculate them using adequate complex refractive indices (the calculation results are shown in Table II in the Appendix). They indicate that the $Q$ factors calculated using complex indices $\left(Q_{\text {total }}\right)$ are significantly smaller than those calculated using real indices. The observed reduction in $Q_{\text {total }}$ is due to free carrier absorption, which is not negligible for QCL devices in the midinfrared region. Nevertheless, the present technique based on angle-resolved reflection makes it possible to determine the output coupling strengths, which are generally nonmeasurable if we use standard spectral techniques.

\section{CONCLUSIONS}

In conclusion, we propose angle-resolved reflection measurement with using a Fourier-transform spectrometer as a useful technique with which to determine the inplane dispersion relations of 2D PC slabs in the midinfrared region. Polarization analysis enables us to assign all the complex PC modes. The peak intensity evaluation enables us to determine the mode $Q$ factors. Thus,

\section{(a)}

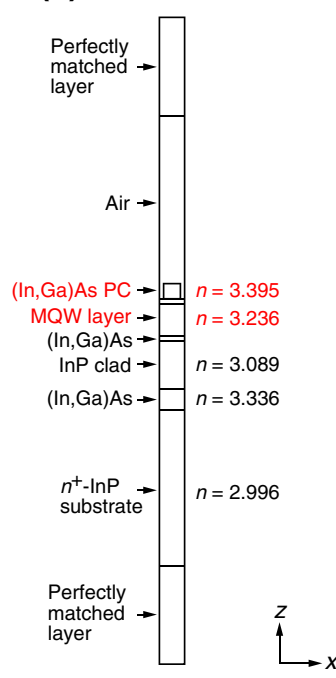

(b) $\mathrm{TE}^{(1)}$

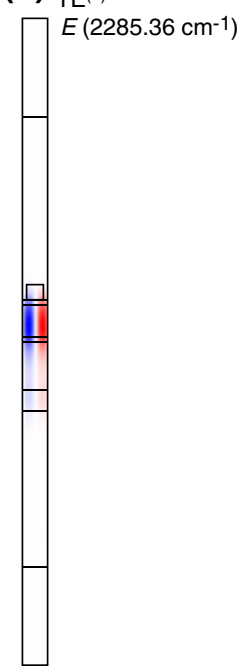

(c) $\mathrm{TE}^{(2)}$

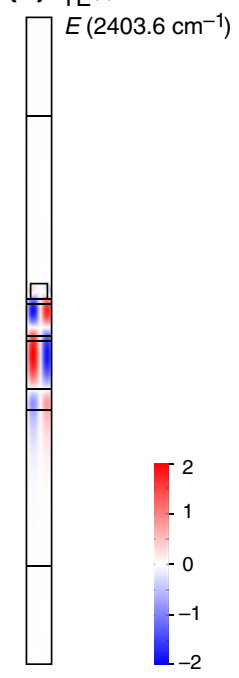

FIG. 5. The vertical field distributions. (a) The layer sequence used for our calculation. Note that the $z$ axis is normal to the slab surface. The refractive index $n$ of each layer is also indicated. The red-colored notations highlight the waveguide core region. (b),(c) The $z$ component of the magnetic field $\left(H_{z}\right)$ for the lowest TE-like mode $\left(\mathrm{TE}^{(1)}\right)$ and the second-lowest TE-like mode $\left(\mathrm{TE}^{(2)}\right)$, respectively. the reflection measurement is useful for determining the optimal conditions for PCSEL structures.

A potential problem inherent in the proposed technique is the limited angular resolution and it might be rather difficult to distinguish fine-splitting spectra at the $\Gamma$ point. However, the problem will be simply overcome by introducing a tunable external-cavity QCL as an excitation source, since it generates a near-plane-wave mid-IR beam. However, the frequency-tunable range is not so large. The adoption of our Fourier spectrometer-based technique for broad spectral analysis and frequency-tunable QCLs for narrower spectral analysis should constitute a valuable approach for fully characterizing PC-slab samples that exhibit sharp resonance spectra.
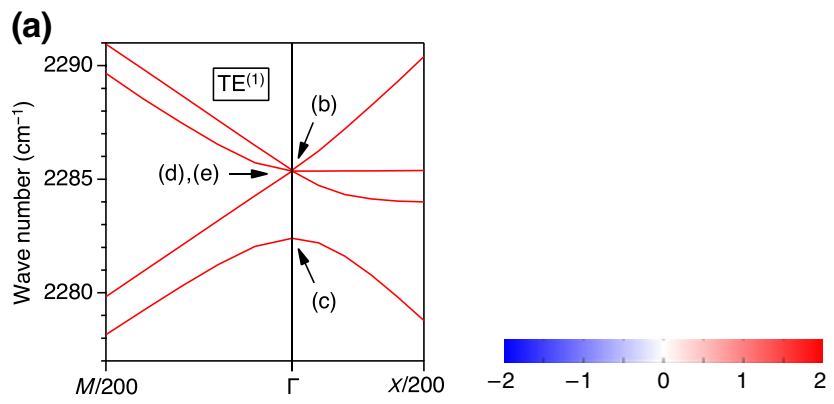

(b)

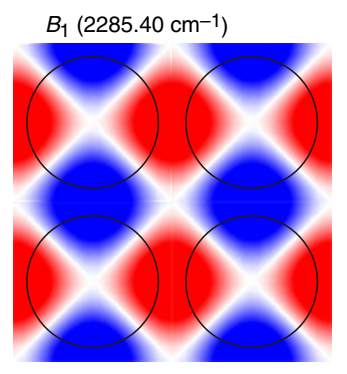

(c)

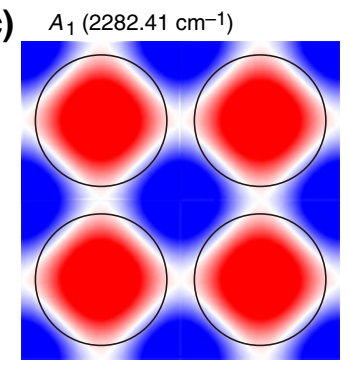

(d)

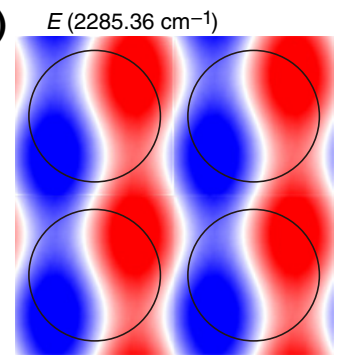

(e)

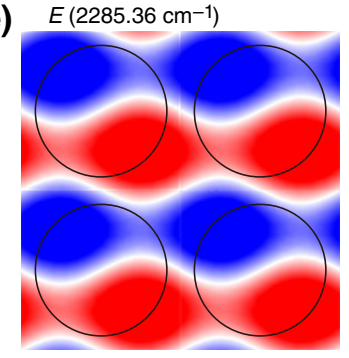

FIG. 6. The in-plane field distributions for the $\mathrm{TE}^{(1)}$ modes at the $\Gamma$ point. (a) The dispersion relations in the vicinity of the $\Gamma$ point. (b)-(e) The $z$ component of the magnetic field $\left(H_{z}\right)$ for modes depicted by the arrows in (a). The black lines indicate the boundaries of circular pillars, which compose PC structures. All the in-plane distributions show their peculiar symmetries, which are characterized by (b) $B_{1}$, (c) $A_{1}$, and (d),(e) $E$ for the $C_{4 v}$ symmetry. Note that the dispersion relations in (a) suggest the degeneracy of $B_{1}$ and $E$ at the $\Gamma$ point and the formation of photonic Dirac cones [19-21], but in fact their eigenfrequencies are slightly different under the model condition. 


\section{ACKNOWLEDGMENTS}

This work was supported by the Innovative Science and Technology Initiative for Security, Grant No. JPJ004596, Acquisition, Technology \& Logistics Agency (ATLA), Japan. S.C. acknowledges support from the National Institute for Materials Science (NIMS) Graduate Assistantship program.

\section{APPENDIX: ANALYSIS OF ELECTROMAGNETIC FIELD DISTRIBUTIONS}

This appendix shows the computed magnetic field distributions of our photonic crystal slab samples. They are calculated using the finite-element method.
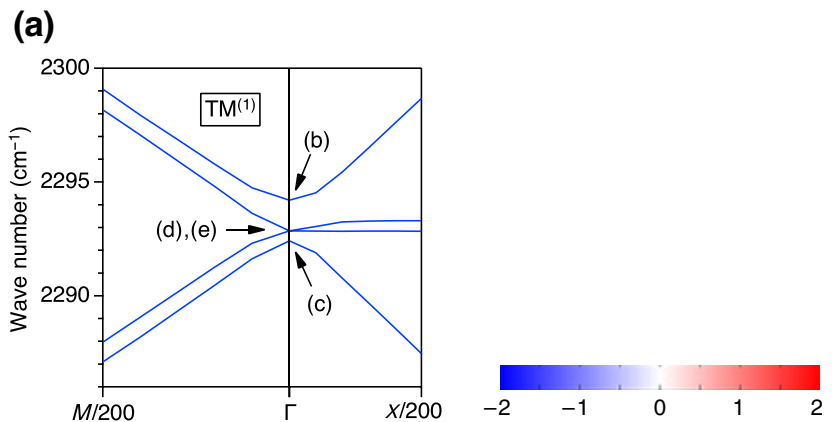

(b)

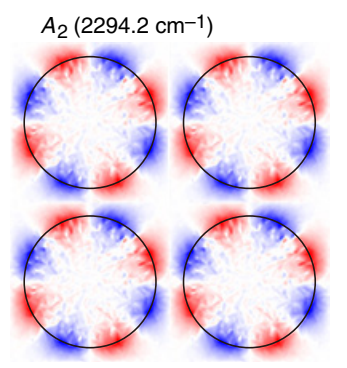

(c)

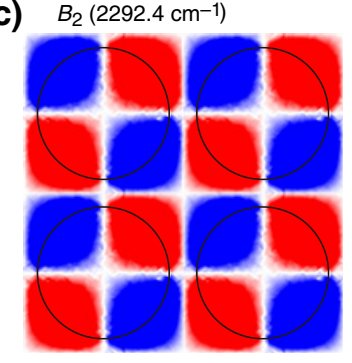

(d)

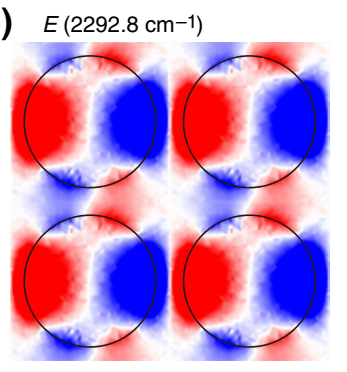

(e)

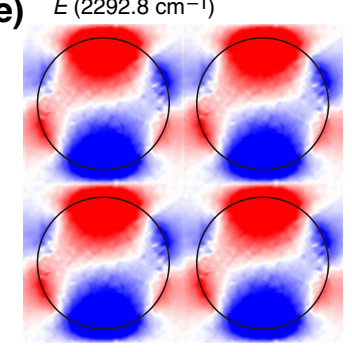

FIG. 7. The in-plane field distributions for the $\mathrm{TM}^{(1)}$ modes at the $\Gamma$ point. (a) The dispersion relations in the vicinity of the $\Gamma$ point. (b)-(e) The $z$ component of the magnetic field $\left(H_{z}\right)$ for modes depicted by the arrows in (a). The black lines indicate the boundaries of circular pillars, which compose PC structures. Note that the relatively large fluctuation of the image data is due to small $H_{z}$ values for the TM-like modes. Nevertheless, we can identify their spatial symmetries, which are characterized by (b) $A_{2}$, (c) $B_{2}$, and (d),(e) $E$ for the $C_{4 v}$ symmetry.
TABLE II. A comparison of the $Q$ factors calculated using real refractive indices and those calculated using complex refractive indices $\left(Q_{\text {total }}\right)$ for each $\mathrm{TE}^{(1)}$ and $\mathrm{TM}^{(1)}$ mode at (a) $k=X / 50$ along the $\Gamma-X$ direction and (b) $k=M / 50$ along the $\Gamma-M$ direction. Note that the $Q$ values calculated using real indices are used to plot Figs. 4(b) and 4(c).

\begin{tabular}{lccc}
\hline \hline & $\begin{array}{c}\text { Wave number } \\
\left(\mathrm{cm}^{-1}\right)\end{array}$ & $\begin{array}{c}Q \\
(\text { real } n)^{\mathrm{a}}\end{array}$ & $\begin{array}{c}Q_{\text {total }} \\
(\text { complex } n)^{\mathrm{b}}\end{array}$ \\
\hline (a) $\Gamma-X(k=X / 50)$ & & \\
$\mathrm{TE}^{(1)} A_{1}$ & 2262.4 & 10968 & 2067 \\
$\mathrm{TM}^{(1)} B_{2}$ & 2270.9 & 2131 & 1131 \\
$\mathrm{TE}^{(1)} E$ & 2284.0 & 915694 & 2621 \\
$\mathrm{TE}^{(1)} E$ & 2285.5 & 5909 & 1788 \\
$\mathrm{TM}^{(1)} E$ & 2293.0 & 1050 & 743 \\
$\mathrm{TM}^{(1)} E$ & 2293.4 & 1064952 & 2489 \\
$\mathrm{TE}^{(1)} B_{1}$ & 2306.9 & 12718 & 2211 \\
$\mathrm{TM}^{(1)} A_{2}$ & 2315.3 & 2087 & 1155 \\
$(\mathrm{~b}) \Gamma-M(k=M / 50)$ & & \\
$\mathrm{TE}^{(1)} A_{1}$ & 2261.8 & 11026 & 2079 \\
$\mathrm{TE}^{(1)} E$ & 2263.3 & 10241 & 2012 \\
$\mathrm{TM}^{(1)} B_{2}$ & 2270.5 & 2124 & 1147 \\
$\mathrm{TM}^{(1)} E$ & 2271.4 & 2198 & 1131 \\
$\mathrm{TE}^{(1)} E$ & 2306.2 & 10689 & 2136 \\
$\mathrm{TE}^{(1)} B_{1}$ & 2307.7 & 11701 & 2159 \\
$\mathrm{TM}^{(1)} E$ & 2314.9 & 2094 & 1174 \\
$\mathrm{TM}^{(1)} A_{2}$ & 2315.8 & 2078 & 1138 \\
\hline \hline
\end{tabular}

${ }^{\text {a }}$ The list of the real $n$ values adopted for our calculation is shown in Fig. 5(a).

${ }^{\mathrm{b}}$ The imaginary parts of the complex $n$ values are estimated based on the doping levels of the QCL samples.

Figure 5 is a comparison between the vertical field distributions of the lowest TE-like mode $\left(\mathrm{TE}^{(1)}\right)$ and the second-lowest TE-like mode $\left(\mathrm{TE}^{(2)}\right)$. Figure 5(a) shows the layer sequence of our sample and the refractive indices used for our calculation. Figure 5(b) shows the field distribution of $\mathrm{TE}^{(1)}$. It is strongly confined in the MQW layer along the $z$ axis, which is normal to the slab surface. Figure 5(d) shows the field distribution of $\mathrm{TE}^{(2)}$, which indicates a nodal distribution along the $z$ axis. See the dispersion relations of $\mathrm{TE}^{(1)}$ and $\mathrm{TE}^{(2)}$ in Fig. 3(b) in the main text and the in-plane field distributions of $\mathrm{TE}^{(1)}$ in Fig. 6(d).

Figure 6 shows the in-plane magnetic field distributions of the TE-like modes and Fig. 7 shows those of the TM-like modes. All the in-plane distributions exhibit their peculiar symmetries, as can be seen in each panel.

[1] M. Imada, S. Noda, A. Chutinan, T. Tokuda, M. Murata, and G. Sasaki, Coherent two-dimensional lasing action in surface-emitting laser with triangular-lattice photonic crystal structure, Appl. Phys. Lett. 75, 316 (1999). 
[2] K. Hirose, Y. Liang, Y. Kurosaka, A. Watanabe, T. Sugiyama, and S. Noda, Watt-class high-power, high-beam-quality photonic-crystal lasers, Nat. Photon. 8, 406 (2014).

[3] S. Noda, K. Kitamura, T. Okino, D. Yasuda, and Y. Tanaka, Photonic-crystal surface-emitting lasers: Review and introduction of modulated-photonic crystals, IEEE J. Sel. Top. Quant. Electron. 23, 1 (2017).

[4] K. Ishizaki, M. De Zoysa, and S. Noda, Progress in photonic-crystal surface-emitting lasers, Photonics 6, 96 (2019).

[5] K. Sakai, E. Miyai, T. Sakaguchi, D. Ohnishi, T. Okano, and S. Noda, Lasing band-edge identification for a surfaceemitting photonic crystal laser, IEEE J. Sel. Areas Commun. 23, 1335 (2005).

[6] D. M. Williams, K. M. Groom, B. J. Stevens, D. T. D. Childs, R. J. E. Taylor, S. Khamas, R. A. Hogg, N. Ikeda, and Y. Sugimoto, Epitaxially regrown GaAs-based photonic crystal surface-emitting laser, IEEE Photonics Technol. Lett. 24, 966 (2012).

[7] R. J. E. Taylor, D. M. Williams, J. R. Orchard, D. T. D. Childs, S. Khamas, and R. A. Hogg, Band structure and waveguide modelling of epitaxially regrown photonic crystal surface-emitting lasers, J. Phys. D: Appl. Phys. 46, 264005 (2013).

[8] V. N. Astratov, R. M. Stevenson, M. S. Skolnick, D. M. Whittaker, S. Brand, I. Culshaw, T. F. Krauss, R. M. De La Rue, and O. Z. Karimov, Experimental technique to determine the band structure of two-dimensional photonic lattices, IEE Proc.-Optoelectron. 145, 398 (1998).

[9] R. Colombelli, K. Srinivasan, M. Troccoli, O. Painter, C. F. Gmachl, D. M. Tennant, A. M. Sergent, D. L. Sivco, A. Y. Cho, and F. Capasso, Quantum cascade surfaceemitting photonic crystal laser, Science 302, 1374 (2003).

[10] C. Boyle, C. Sigler, J. D. Kirch, D. F. Lindberg, T. Earles, D. Botez, and L. J. Mawst, High-power, surface-emitting quantum cascade laser operating in a symmetric grating mode, Appl. Phys. Lett. 108, 121107 (2016).

[11] Y. Liang, Z. Wang, J. Wolf, E. Gini, M. Beck, B. Meng, J. Faist, and G. Scalari, Room temperature surface emission on large-area photonic crystal quantum cascade lasers, Appl. Phys. Lett. 114, 031102 (2019).
[12] Z. Wang, Y. Liang, B. Meng, Y.-T. Sun, G. Omanakuttan, E. Gini, M. Beck, I. Sergachev, S. Lourdudoss, J. Faist, and G. Scalari, Large area photonic crystal quantum cascade laser with $5 \mathrm{~W}$ surface-emitting power, Opt. Express 27, 22708 (2019).

[13] Y. Yao, N. Ikeda, T. Kuroda, T. Mano, H. Koyama, Y. Sugimoto, and K. Sakoda, Mid-IR Dirac-cone dispersion relation materialized in SOI photonic crystal slabs, Opt. Express. 28, 4194 (2020).

[14] T. Kuroda, S. Chalimah, Y. Yao, N. Ikeda, Y. Sugimoto, and K. Sakoda, Apparatus for high-precision angle-resolved reflection spectroscopy in the mid-infrared region, Appl. Spectrosc. 75, 259 (2021).

[15] K. Sakoda, Optical Properties of Photonic Crystals (Springer-Verlag, Berlin, 2004), 2nd ed.

[16] S. Saito, R. Hashimoto, K. Kaneko, T. Kakuno, Y. Yao, N. Ikeda, Y. Sugimoto, T. Mano, T. Kuroda, H. Tanimura, S. Takagi, and K. Sakoda, Surface-emitting quantum cascade laser with strain-compensated quantum wells and photonic crystals (unpublished 2021).

[17] Y. Yao, T. Kuroda, N. Ikeda, T. Mano, H. Koyama, Y. Sugimoto, and K. Sakoda, Angle-resolved reflection spectra of Dirac cones in triangular-lattice photonic crystal slabs, Opt. Express 28, 21601 (2020).

[18] K. Ohtaka, Y. Suda, S. Nagano, T. Ueta, A. Imada, T. Koda, J. S. Bae, K. Mizuno, S. Yano, and Y. Segawa, Photonic band effects in a two-dimensional array of dielectric spheres in the millimeter-wave region, Phys. Rev. B 61, 5267 (2000).

[19] X. Huang, Y. Lai, Z. H. Hang, H. Zheng, and C. T. Chan, Dirac cones induced by accidental degeneracy in photonic crystals and zero-refractive-index materials, Nat. Mater. 10, 582 (2011).

[20] K. Sakoda, Proof of the universality of mode symmetries in creating photonic Dirac cones, Opt. Express. 20, 25181 (2012).

[21] S. Chalimah, Y. Yao, N. Ikeda, Y. Sugimoto, T. Mano, T. Kuroda, and K. Sakoda, Eigenmode symmetry assignment of triangular-lattice photonic crystal slabs and their Dirac cones materialized by effective degeneracy in the mid-infrared region, Opt. Express 29, 19486 (2021). 